\title{
A regional-scale survey to define the known and potential vectors of grapevine yellow phytoplasmas in vineyards South of Swiss Alps
}

\author{
Valeria Trivellone - Luisa Filippin • \\ Barbara Narduzzi-Wicht • Elisa Angelini
}

Accepted: 4 February 2016/Published online: 12 February 2016

(C) Koninklijke Nederlandse Planteziektenkundige Vereniging 2016

\begin{abstract}
The most important Grapevine Yellows (GY) phytoplasma diseases in Europe are Flavescence dorée (FD) and Bois noir (BN); they are spread in vineyard by two proven vectors, Scaphoideus titanus Ball and Hyalesthes obsoletus Signoret, respectively. Other potential vectors of GY have been identified, which are thought to play a secondary role. The GY control strategies are not always effective and an in-depth study on the ecological cycle of the pathogens at regional scale would be of paramount importance. This study was carried out in 48 representative sites of wine-growing area South of Swiss Alps, with the aim to identify known and potential
\end{abstract}

Electronic supplementary material The online version of this article (doi:10.1007/s10658-016-0880-3) contains supplementary material, which is available to authorized users.

V. Trivellone $(\bowtie)$

Station de recherche Agroscope Changins-Wädenswil ACW, Cadenazzo, Switzerland

e-mail: valeria.trivellone@gmail.com

L. Filippin · E. Angelini

CREA-VIT Centro di Ricerca per la Viticoltura, Conegliano (TV), Italy

B. Narduzzi-Wicht

Laboratory of Applied Microbiology, DECD, SUPSI, Bellinzona, Switzerland

V. Trivellone

Swiss Federal Research Institute WSL, Birmensdorf, Switzerland

V. Trivellone

Laboratory of Soil Biology, University of Neuchâtel, Neuchâtel, Switzerland vectors and to characterize the FD and BN phytoplasmas isolates. Out of 167 Auchenorrhyncha species recorded, 27 were known or potential vectors of phytoplasmas and five of those tested positive for phytoplasmas. S. titanus was infected by $16 \mathrm{SrV}-\mathrm{D}$ subgroup phytoplasma and no clear relationship between its population density and disease outbreaks was observed. Orientus ishidae harboured $16 \mathrm{SrV}-\mathrm{C}$ and $16 \mathrm{SrV}-\mathrm{D}$ subgroups suggesting its potential role in spreading $16 \mathrm{SrV}-\mathrm{C}$ phytoplasma isolates from arboreal plants to grapevine and FD-D from grapevine to grapevine. $H$. obsoletus was infected by $\mathrm{BN}$ phytoplasmas, tuf-types a and b, however it was collected with relatively low abundance. Reptalus panzeri and $R$. cuspidatus tested positive to tuf-type b, but only $R$. cuspidatus was common and abundant in the investigated vineyards. To define the range of alternative vectors using a detailed approach on regional scale provides background information to get a more clear vision on the spreading of phytoplasmas in the vineyards.

Keywords Auchenorrhyncha - Grapevine yellows . Molecular detection $\cdot$ Vectors

\section{Introduction}

Grapevine yellows (GY) are severe diseases occurring in Vitis vinifera in all wine growing countries. They cause very serious damage in viticulture and wine industry, ranging from a lower yield of berries and wine to the progressive decline and death of the infected plants. The etiological agents of GY are phytoplasmas, belonging to 
the new-established genus 'Candidatus Phytoplasma' ( $\mathrm{Ca}$. P.) that includes at least 37 different species and phylogenetic groups (IRPCM, Phytoplasma/Spiroplasma Working Team-PhytoplasmaTaxonomy Group 2004; Marcone 2014). Only some phytoplasma species occur in grapevine worldwide, causing similar but distinct diseases.

In Europe, two phytoplasmas are typically present in grapevine: phytoplasmas belonging to the $16 \mathrm{SrV}-\mathrm{C}$ and 16SrV-D phylogenetic subgroups, causing Flavescence dorée (FD), and 'Ca. P. solani' (stolbur phytoplasma), belonging to the 16SrXII phylogenetic group and associated to Bois noir (BN). FD is a quarantine disease in the European Community, discovered in France in the '70s and nowadays occurring in several countries of Southern and Central Europe (EPPO 2007). Different FD phytoplasma isolates were identified in grapevine, such as FD70, FD-D (=FD92) and FD-C, they are transmitted from vine to vine by Scaphoideus titanus Ball (Schvester et al. 1963), a nearctic leafhopper that spends its whole life cycle on Vitis spp. and disseminates FD phytoplasmas in an epidemical manner. BN phytoplasma is transmitted by the occasional grapevinefeeder Hyalesthes obsoletus Signoret that usually lives on weeds (Sforza et al. 1998; Maixner 1994). BN is spread in Europe and in the Mediterranean Basin, where it generally shows an endemic behaviour. Indeed ' $\mathrm{Ca}$. $\mathrm{P}$. solani' usually infects only a low percentage of the plants in vineyard, due to the different ecological cycle of the pathogen. According to the genetic sequences of the elongation factor Tu, two main ' $\mathrm{Ca}$. P. solani' types can be distinguished, tuf-type a and tuf-type $\mathrm{b}$, which are involved in two different epidemiological cycles mainly related to H. obsoletus (Langer and Maixner 2004). Tuftype a is associated to Urtica dioica and tuf-type b to Convolvulus arvensis. Other known and potential vectors of GY have been recognized in Europe, which are thought to play a secondary role in GY epidemiology. In Germany, the alder-feeding Hemiptera Oncopsis alni (Schrank) (Maixner and Reinert 1999) was demonstrated to transmit PGY (Palatinate Grapevine Yellows) phytoplasma, similar to FD phytoplasma, from the black alder, Alnus glutinosa, to grapevine (Maixner et al. 2000). In Italy and Serbia the polyphagous planthopper Dictyophara europaea (Linnaeus) was shown to be able to transmit FD phytoplasma from wild clematis, Clematis vitalba, to grapevine (Filippin et al. 2009). Orientus ishidae (Matsumura) was found infected by FD and FD-related phytoplasmas in a few countries, thus it is considered a suspected vector (Mehle et al.
2010; Gaffuri et al. 2011). Naturally infected Reptalus panzeri (Low) specimens were able to transmit ' $\mathrm{Ca}$. $\mathrm{P}$. solani' to grapevine in Serbia (Cvrković et al. 2014), and the same phytoplasma was identified in other Reptalus species (Pinzauti et al. 2008; Palermo et al. 2004; Mikec et al. 2006). At last, other leafhopper species inhabiting vineyard agroecosystems were found to harbour ' $C a$. P. solani', such as Euscelis lineolatus Brullé, Anaceratagallia ribauti (Ossiannilsson) and Macrosteles quadripunctulatus (Kirschbaum), but their ability to transmit BN phytoplasma to grapevine has not been confirmed (Batlle et al. 2008; Riedle-Bauer et al. 2008; Landi et al. 2013).

GY control strategies are mainly focused on prevention, survey and insecticide treatments, which are not always effective. Concerning FD, despite mandatory control programs against $S$. titanus and grubbing of diseased vineyards, the contaminated surfaces increase every year, suggesting that the epidemiology of the diseases is still not completely understood. In the case of $\mathrm{BN}$, there are no established and effective control strategies, as the insecticide treatments are useless in vineyards, due to the fact that the vector feeds only occasionally on grapevine. Indeed, occurrence of $\mathrm{BN}$ has increased over the last few years in most European countries (Johannesen et al. 2008).

Against this background, an in-depth study on the ecological cycle of the pathogens, together with the research of alternative vectors and their role in GY outbreaks, would be of paramount importance, because the epidemiological context could be quite different in diverse countries.

In Switzerland BN and FD diseases have been recorded in 2001 (Schmid and Emery 2001) and 2004 (Schaerer et al. 2007), respectively; however the epidemiological aspects are still poorly understood. BN is quite widespread in all wine-growing regions, while FD occurs only South of the Swiss Alps. A number of actions have already been undertaken in order to cope with the diffusion of known vectors; nevertheless, so far, outbreaks of GY are still observed. The objectives of the present work were: 1) to identify known and potential vectors by means of a detailed screening of leafhoppers inhabiting southern Swiss vineyards; 2) to assess the presence of $\mathrm{FD}$ and $\mathrm{BN}$ phytoplasmas in the insects captured in GY infected vineyards; and 3) to characterize the phytoplasma isolates infecting leafhoppers. In this context, we also discuss the use of an experimental design based on a representative subset of samples to address phytoplasma disease issues. To this aim, a 
representative sample of vineyards south of the Swiss Alps was surveyed for the Auchenorrhyncha and phytoplasma occurrence, in order to gain more insight and to lay the groundwork for further investigations.

\section{Materials and methods}

\section{Study area and experimental design}

The study area comprises the whole wine-growing area of southern Switzerland which extends from the northernmost site Ludiano $\left(46^{\circ} 25^{\prime} \mathrm{N}-8^{\circ} 58^{\prime} \mathrm{E}\right)$ to the southernmost site Pedrinate $\left(45^{\circ} 49^{\prime} \mathrm{N}-9^{\circ} 00^{\prime} \mathrm{E}\right)$, ranging from $199 \mathrm{~m}$ to $589 \mathrm{~m}$ a.s.l.

Forty eight study sites were selected using a design that accounted for the three main variables characterizing the vineyard agroecosystem of the study region, i.e. aspect (24 sites were exposed SE-SW; 24 sites NE-NW), slope (24 sites were on a plain $<5^{\circ} ; 24$ sites were terraced $>10^{\circ}$ ) and the dominant land use type ( $>50 \%$ land unit cover) surrounding the vineyard within a radius of $500 \mathrm{~m}$ (16 sites were dominated by forest, 16 by settlements, 16 by open areas). The 48 selected vineyards could be considered representative of the vineyard ecosystem and landscape of southern Switzerland (Supplementary material 1).

Since 2004, a surveillance monitoring was launched by the phytosanitary office in southern Switzerland in order to oversee the spread of phytoplasma diseases (Jermini et al. 2014). Out of 48 investigated vineyards, five were affected by FD, 13 by BN, 10 by both and 20 showed no GY symptoms in the last decade. A chitin synthesis inhibitor insecticide (Buprofezin) was applied twice per year (on June) in 26 studied vineyards (Supplementary material 1).

In the study area, the vineyard floor is usually permanently covered by wild native plants, e.g. Trifolium spp., Plantago lanceolata, Stellaria media, Erigeron annuus, Ranunculus spp. (for further details on the floristic composition of vineyards of this study see Trivellone et al. 2014). In some vineyards, the vine rows could be tilled or treated with herbicides (a strip of around $50 \mathrm{~cm}$ of width below the grapevines).

Collection and faunistic data evaluation

In 2011, multiple sampling methods were used to effectively pick up the greatest number of specimens and species of leafhoppers. Four standard methods were selected: D-Vac sampler, pitfall traps, beating tray and yellow sticky traps. The first two were used for capturing leafhoppers related to the herb layer and to the soil surface, the last two for the species inhabiting the vine canopy. Vineyards were sampled from April to October, for a total of seven sampling periods. In each vineyard two sampling sites were placed which consisted of a pitfall trap and a sticky trap, one site along a vine row and the other in a vegetated embankment (in terraced vineyards) or along another vine row (in lowland vineyards). The two sampling sites were at least $20 \mathrm{~m}$ apart and $20 \mathrm{~m}$ away from vineyard margin to avoid edge effects; the traps were open for seven days per month in each sampling period. The pitfall trap site consisted in four $200 \mathrm{ml}$ cups ( $7 \mathrm{~cm}$ of diameter) recessed into the soil and arranged along a vine row or embankment, and spaced about $1 \mathrm{~m}$ one another. Each cup was half-filled with saline solution and covered by a transparent plastic roof. The yellow sticky trap $\left(\right.$ Rebell $^{\circledR}, 15 \times 8 \mathrm{~cm}$ dimensions) was hung inside the vine canopy at about $1.5 \mathrm{~m}$ above the ground. By means of D-vac sampler and beating tray, two samples were collected monthly from two transects inside the vineyard. The D-Vac sampler was applied on ground cover vegetation during $120 \mathrm{~s}$ per sample. Beating tray (sheet opening $1 \times 1 \mathrm{~m}$ ) was applied to collect all arthropods that fell down from vine canopy after shaking of thirty grapevines per sample. Additional sampling sites were placed along the vineyard-forest ecotone in a total of 16 vineyards (Supplementary material 1).

All collected leafhopper specimens were identified to species level, then preserved in $70 \%$ alcohol and maintained at $-20^{\circ} \mathrm{C}$. Nomenclature followed Ribaut (1936); Della Giustina (1989) and Holzinger et al. (2003).

Sample-based rarefaction curve was calculated using vineyard as the sample to evaluate sampling adequacy to detect the regional (gamma) species richness (Gotelli and Colwell 2001).

After a literature review and field data evaluation, a sub-group of Auchenorrhyncha species known or potential vectors of phytoplasmas were selected for the investigated region; the relationship between species mean abundance and species occurrence was examined and the specimens were subjected to molecular analyses.

\section{DNA extraction and amplification}

Each sample consisted of a pool of up to 20 specimens of the same species. Total DNA was extracted according to Gatineau et al. (2001). 
TaqMan real time PCR analysis on ribosomal genes was carried out to identify the presence of the $16 \mathrm{SrV}$ and 16SrXII group phytoplasmas, according to the protocols already described (Angelini et al. 2007). The assays were performed in 96-well plates on a CFX96 thermal cycler (Biorad). Positive samples were then amplified by nested PCR on three different DNA fragments for a deeper genetic characterization. ' $C a$. $P$. solani' positive samples were analysed in the $16 S-23 S$ ribosomal $R N A$, tuf and $\sec Y$ nucleotide regions. FD phytoplasmas positive samples were screened in the $16 \mathrm{~S}-23 \mathrm{~S} \mathrm{rRNA}$, rplV$\operatorname{rps} C$ and $\sec Y$ regions.

In the $16 S$-23S rRNA region, the first PCR was performed using universal primer pair $\mathrm{P} 1 / \mathrm{P} 7$ and the nested PCR with primers $16 \mathrm{r} 758 \mathrm{f} / \mathrm{M} 23 \mathrm{Sr}$ (Angelini et al. 2001).

FD phytoplasmas positive samples were amplified in the $r p l V-r p s C$ region, encoding for ribosomal proteins L22 and S3, in nested PCR using primers $r p(V) F 1 / r p R 1$, followed by $\mathrm{rp}(\mathrm{V}) \mathrm{F} 1 \mathrm{~A} / \mathrm{rp}(\mathrm{V}) \mathrm{R} 1 \mathrm{~A}$ (Martini et al. 2002; Lee et al. 2004). PCR assays on the $\sec Y$ gene, encoding the preprotein translocase subunit $\mathrm{SecY}$, were performed with primers FD9f2/FD9r, followed by FD9f3/FD9r2 (Angelini et al. 2001). The concentrations of reagents and PCR conditions for amplification of $16 S-23 S r R N A$, $r p l V$-rpsC and sec $Y$ regions were as described in Angelini et al. (2001) and Martini et al. (2002).

Characterization of ' $C a$. P. solani' was performed in the tuf gene using primers fTufl/rTuf1, followed by fTufAy/rTufAy, according to Schneider et al. (1997). Tuf gene encodes for elongation factor Tu. For the secY gene of ' $C a$. $P$. solani' positive samples, new primers were designed, using the rplO-secY-adk sequences of three phytoplasmas phylogenetically close to 'Ca. P. solani': two ' $\mathrm{Ca}$. Phytoplasma asteris' isolates (AYWB Aster yellows witches'-broom phytoplasma, GenBank accession number CP000061, and OY-M Onion yellows phytoplasma, GenBank accession number AP006628) and one ' $\mathrm{Ca}$. Phytoplasma australiense' isolate (GenBank accession number AM422018). This genomic region codifies for the $50 \mathrm{~S}$ ribosomal protein $\mathrm{L} 15$, the preprotein translocase subunit $\mathrm{SecY}$ and the Adenylate kinase. The forward primer RPLOf2 (5'CAA AGA ATT CCT AAA AGA GG-3') and the reverse ADKr2 (5'-GCT TGA GTG CCT TTG CCA ATT CC-3') were used for the direct PCR, whereas the pair RPLOf3 (5'-TCT ATT TTA GCA GTT GGT GG$3^{\prime}$ ) and BN9r0 (5'-AAA CTT GTT CCT CCT AAT TTC-3') was used for the nested PCR. The reaction mixture contained $1 \mu \mathrm{l}$ of extracted DNA or of the diluted first PCR product (1:50) as template, $0.3 \mathrm{mM}$ each dNTP, $0.6 \mu \mathrm{M}$ each primer, $0.75 \mathrm{U}$ Taq DNA polymerase (Sigma Aldrich) and the buffer supplied with the enzyme. The $\mathrm{MgCl}_{2}$ concentrations were $3 \mathrm{mM}$ and $1.5 \mathrm{mM}$ in the direct and nested PCR, respectively. The following thermal protocol was used for both amplifications: initial denaturation at $94{ }^{\circ} \mathrm{C}$ for $3^{\prime}$, then 40 cycles of $1^{\prime}$ at $94^{\circ} \mathrm{C}, 2^{\prime}$ at $48^{\circ} \mathrm{C}$ and $3^{\prime}$ at $66^{\circ} \mathrm{C}$, and a final extension for $7^{\prime}$ at $66^{\circ} \mathrm{C}$. The final PCR products are $1202 \mathrm{bp}$ long.

Amplicons were visualized on $1 \%$ agarose gel stained with GelRed (Biotium Inc.) under a GelDoc XR UV transilluminator (Biorad).

\section{RFLP analysis and DNA sequencing}

Aliquots of the nested PCR products obtained from the 16S-23S rRNA and tuf genomic regions were digested with restriction enzymes, according to the manufacturer's instructions. The 16r758f/M23 Sr fragments were processed using TaqI (MBI Fermentas), the fTufAy/ rTufAy fragments using HpaII (MBI Fermentas). The restriction patterns were compared with those of FD-D, FD-C and ' $C a$. P. solani' phytoplasmas for $16 S-23 S$ $r R N A$ amplicons, and with a tuf-type a and a tuf-type $\mathrm{b}$ BN phytoplasmas for tuf ones. Restriction products were separated by $10 \%$ polyacrylamide gel electrophoresis in TBE buffer, stained with GelRed and visualized with GelDoc XR.

All amplicons were purified with Sephadex G100 (GE Healthcare). Quantification was carried out with a ND-100 Spectrophotometer (NanoDrop Technologies Inc.). Sequencing was performed with the BigDye Terminator 3.1 Cycle Sequencing Kit (Applied Biosystems) and the products were purified with Sephadex G-50 (Sigma-Aldrich). Samples were finally loaded into an automatic ABI PRISM 3130xl Genetic Analyzer (Applied Biosystems).

Sequence analysis

Electropherograms were corrected and aligned using CLUSTAL W with BioEdit 7.0.9. Phylogenetic trees were constructed with the Maximum Parsimony (MP), Minimum Evolution (ME) and Neighbor Joining (NJ) methods using the MEGA 5.0 software package. The reliability of the analyses was subjected to a bootstrap test with 1000 replicates. Reference sequences were 
selected from GenBank. A BLAST query was previously performed in order to select the most similar reference sequences. Nucleotide sequences obtained in this study were deposited in the DDJB/EMBL/GenBank databases under accession numbers KP635226-KP635235, KT310178 (for BN secY gene), KR350639KR350642, KT371524- KT371527 (for FD secY gene), KP890031, KP941109, KP941110, KR024255KR024261(for 16S-23S rRNA region), KR029136KR029140 (for tuf gene) and KR350643-KR350645, KT371528- KT371531 (for rplV-rpsC region) (Supplementary material 2).

\section{Results}

Occurrence of Auchenorrhyncha known and potential vectors

In total, 60936 specimens belonging to 167 Auchenorrhyncha species were recorded from the 48 sites across four sampling methods (Supplementary material 3). Among them, there were 39 Fulgoromorpha species from the families Cixiidae (5), Delphacidae (28), Dictyopharidae (1), Flatidae (1), Issidae (2), Tettigometridae (2), and 128 Cicadomorpha species from the families Aphrophoridae (5), Cercopidae (2), Membracidae (3), Ulopidae (1), Cicadellidae (117). The supplementary Figure 4 illustrates the rarefaction curve based on the number of sampled vineyards for species richness of Auchenorrhyncha fauna: the curve reached an asymptote well before total accumulated sampling effort, suggesting that species inventory was complete in this study.

After a literature review, a total of 22 species out of 167 recorded were allocated to the status of known or putative vectors of phytoplasmas and then suspected to be involved in the spread of phytoplasmas in vineyards in southern Switzerland. After field data evaluation, five further species (i.e. Arocephalus longiceps, Centrotus cornutus, Issus coleoptratus, Japananus hyalinus and Penthimia nigra) were selected and allocated to the status of suspected vectors in this study. These species were considered because are widespread in vineyards where outbreaks of GY were observed and no presence of known or potential vectors, according to literature, was recorded (Supplementary material 5). Overall, 27 species were considered for molecular analyses: six of them (A. ribauti, A. longiceps, Macrosteles cristatus,
Psammotettix confinis, Reptalus cuspidatus and S. titanus) were widespread throughout the study area with highest population densities (occurrence $>24$ and mean abundance $>60$ ); five species (Aphrodes makarovi, Euscelis incisus, H. obsoletus, Megophthalmus scanicus and Philaenus spumarius) were also quite widespread but with relatively lower mean abundance (occurrence $>34$ and mean abundance $<30$ ). Macrosteles viridigriseus was much less widespread, but abundant (if not dominant) when present (occurrence $=12$ and mean abundance $=100$ ). Most species (15 as a total) only occurred in vineyard at very low mean abundance and were less widespread (occurrence $\leq 20$ and mean abundance $\leq 20$ ) (Supplementary Figure 6).

Phytoplasma detection and RFLP analyses

PCR analysis was performed on 3529 specimens belonging to 27 species, pooled in 371 samples (Supplementary material 7). Phytoplasma DNA was detected in 19 samples out of 371 (5.1\%), collected from eight vineyards out of $48(17 \%)$, corresponding to five species: $O$. ishidae, $S$. titanus, $H$. obsoletus, $R$. cuspidatus and $R$. panzeri.

The FD and FD-related phytoplasmas were harboured by two out of $149(1.3 \%)$ collected $S$. titanus samples and six out of $22(27 \%)$ collected $O$. ishidae samples. The RFLP analyses of the $16 S-23 S$ rRNA genetic region showed that $O$. ishidae and $S$. titanus harboured FD-related phytoplasmas; notably, all isolates from $S$. titanus samples and one from $O$. ishidae belonged to $16 \mathrm{SrV}-\mathrm{D}$ subgroup, while the other samples from $O$. ishidae belonged to $16 \mathrm{SrV}-\mathrm{C}$ phylogenetic subgroup (Table 1).

The stolbur phytoplasma was harboured by eight $H$. obsoletus samples out of $39(21 \%)$ collected, two $R$. cuspidatus samples out of $28(7 \%)$ and one $R$. panzeri out of seven (14\%). HpaII digestion of tuf amplicons showed a prevalence of tuf-type a in phytoplasmas from H. obsoletus (seven on eight tested positive samples), while one phytoplasma isolate from $\mathrm{H}$. obsoletus and all isolates from Reptalus spp. were tuf-type b (Table 1).

Multilocus sequence typing analyses on FD phytoplasma

To further characterize the detected phytoplasmas, a multilocus sequence typing (MLST) was performed. 
Table 1 Results of phytoplasma strain differentiation by RFLP analyses on $16 S-23 S r R N A$ and tuf amplicons for leafhopper samples tested positive in this study. Details about vineyards are reported in Supplementary material 1

\begin{tabular}{|c|c|c|c|c|}
\hline Insect species & Phytoplasma type & Vineyard & $\begin{array}{l}\text { Local scale } \\
\text { positive/tested (\%) }\end{array}$ & $\begin{array}{l}\text { Regional scale } \\
\text { positive/tested (\%) }\end{array}$ \\
\hline Scaphoideus titanus & 16SrV-D & $\begin{array}{l}\text { Camorino } \\
\text { Stabio }\end{array}$ & $\begin{array}{l}1 / 4(25) \\
1 / 1(100)\end{array}$ & 2/149 (1) \\
\hline \multirow[t]{2}{*}{ Orientus ishidae } & $\begin{array}{l}16 \mathrm{SrV}-\mathrm{D} \\
16 \mathrm{SrV}-\mathrm{C}\end{array}$ & $\begin{array}{l}\text { Stabio } \\
\text { Stabio }\end{array}$ & $\begin{array}{l}1 / 7(14) \\
3 / 7(43)\end{array}$ & $6 / 22(27)$ \\
\hline & & $\begin{array}{l}\text { Lamone } \\
\text { Rancate }\end{array}$ & $\begin{array}{l}1 / 2(50) \\
1 / 1(100)\end{array}$ & \\
\hline \multirow[t]{4}{*}{ Hyalesthes obsoletus } & 16SrXII-A (tuf-type a) & $\begin{array}{l}\text { Rancate } \\
\text { Croglio }\end{array}$ & $\begin{array}{l}1 / 2(50) \\
4 / 10(50)\end{array}$ & $8 / 39(21)$ \\
\hline & & Porza & $1 / 1(100)$ & \\
\hline & & Camorino & $1 / 2(50)$ & \\
\hline & 16SrXII-A (tuf-type b) & Rovio & 1/7 (14) & \\
\hline Reptalus panzeri & 16SrXII-A (tuf-type b) & Besazio & $1 / 1(100)$ & $1 / 7(14)$ \\
\hline Reptalus cuspidatus & 16SrXII-A (tuf-type b) & $\begin{array}{l}\text { Rovio } \\
\text { Croglio }\end{array}$ & $\begin{array}{l}1 / 3(33) \\
1 / 5(20)\end{array}$ & $2 / 28(7)$ \\
\hline
\end{tabular}

Different phylogenetic trees were created for each genetic region. All the trees were constructed using the MP method; the NJ and ME trees generally showed the same topology (data not shown). The $16 S-23 S$ rRNA, rplV-rpsC and $\sec Y$ regions from FD and FD-related phytoplasmas were sequenced, obtaining a total of 23 sequences.

16S-23S rDNA Data from the $r R N A$ genetic fragments always confirmed the RFLP patterns. All 16SrV-D sequences were identical to one another and to the FD-D reference strain (AJ548787), as expected. Also all $16 \mathrm{SrV}-\mathrm{C}$ sequences were identical to one another and to the reference strains (AF458378; AF176319; AF458379; Y16387).

rplV-rpsC The phylogenetic tree based on 769 bp of the $r p l V$-rps $C$ region defined four main groups, each encompassing members of widely used reference strains: FD70, FD-D, FD-C, and ALY (Supplementary Figure 8). The sequence obtained from the sample Oi-63 was not included in this phylogenetic tree because it showed many nucleotide ambiguities, suggesting the presence of at least two distinct $16 \mathrm{SrV}$ isolates. In the first group, three $16 \mathrm{SrV}-\mathrm{C}$ phytoplasma isolates harboured by $O$. ishidae (Oi-46, Oi-370 and Oi-78) showed a sequence identity of $100 \%$ with the French FD70 reference strain, CL-AL31 isolate from an Italian clematis and V04-11-55 isolate from a French grapevine. The phytoplasma isolate from one $O$. ishidae sample (Oi-369) showed a single point mutation with respect to FD70; however, it subgrouped with samples previously collected from grapevines in Northern Italian regions Valle d'Aosta ( VvAO262) and Piedmont (VI04-248-04). In the second group, the Swiss $16 \mathrm{SrV}-\mathrm{D}$ phytoplasma isolates were all identical to the FD-D reference isolate isolated from $V$. vinifera in Italy. The last two groups encompassed reference strains only, represented by FD-C and ALY respectively, which were included just to show the phylogenetic distance from the samples of the present study.

$\sec Y$ The phylogenetic tree based on $1004 \mathrm{bp}$ of $\sec Y$ gene (Fig. 1) showed a similar overall topology to that inferred from the ribosomal protein genes. Some further differentiations are highlighted in the first group where Oi-370, Oi-46 and Oi-78 are slightly different from the FD70 reference strain. In the second group, the three samples Oi-368, St-371, St-182 again showed a $100 \%$ sequence identity with that of FD-D reference strain isolated from $O$. ishidae in Slovenia.

As a total, three vineyards hosted populations of $O$. ishidae infected by the three different isolates of FD and FD-related phytoplasmas. In particular, four out of six $O$. ishidae samples testing positive in this study were collected in a vineyard (Stabio) very close to the border with Lombardy (Italy) where the detected $O$. ishidae population, inhabiting the forest surrounding the vineyard, harboured three different FD variants, belonging 


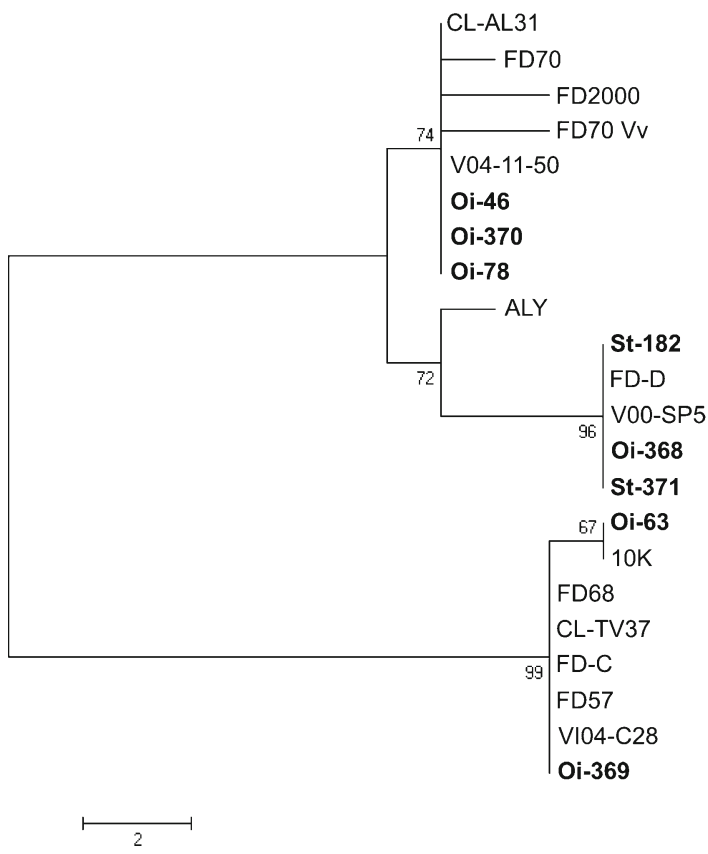

Fig. 1 Phylogenetic tree based on 1004 bp of the $\sec Y$ gene sequences obtained from Scaphoideus titanus and Orientus ishidae samples. Swiss samples processed in the current study are evidenced in bold. Bar length is proportional to the number of base substitutions per site. Numbers on the branches are confidence values obtained for

to $16 \mathrm{SrV}-\mathrm{C}$ and D phylogenetic subgroups. The two $S$. titanus samples (St-371 and St-182), harbouring the same identical isolate, were collected in two different vineyards far away to each other and the insecticides were applied only in one of them (Stabio).

In Table 2 an overview of FD phytoplasma isolates detected in this study is reported.

MLST analyses on stolbur phytoplasma

A MLST analysis was performed on the detected stolbur phytoplasmas. Different phylogenetic trees were created for each genetic region. All the trees were constructed using the MP method; the NJ and ME trees generally showed the same topology (data not shown). The $16 S$ 23S rRNA, tuf and secY nucleotide fragments of stolbur phytoplasmas were sequenced, for a total of 33 nucleotide sequences.

16S-23S $r D N A$ The sequence data of $r R N A$ genomic fragments always confirmed the RFLP patterns. The newly obtained sequences were very similar to one another, showing two or three single nucleotide
Country, Region - Municipality

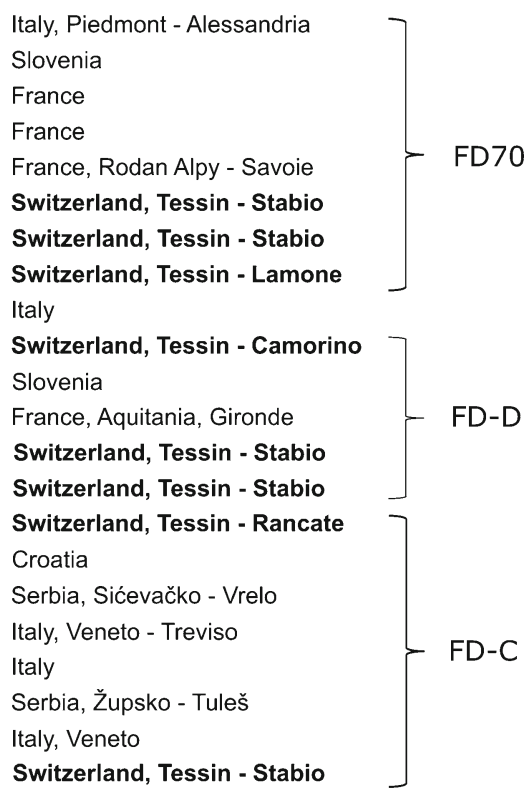

1000 replicates. GenBank accession numbers and details of the reference phytoplasma strains are listed in Supplementary material 2. Host: Ag Alnus glutinosa; $\mathrm{Cv}$ Clematis vitalba; Oi Orientus ishidae; St Scaphoideus titanus; Vv Vitis vinifera

polymorphisms compared to ' $\mathrm{Ca}$. $P$. solani' type strain (AF248959) (data not shown).

tuf The phylogenetic tree based on 826 bp of the tuf gene allowed distinguishing two main groups that correspond to $t u f$-type a and tuf-type $\mathrm{b}$ restriction patterns (Supplementary Figure 9). All H. obsoletus but one (Ho-98) were infected by tuf-type a, whereas Reptalus species by tuf-type b. The sequences of the Swiss samples were identical to one another and to those of many other isolates from grapevines, weeds and insects collected in Central-Eastern Europe. In the tuf-type b cluster, the two $R$. cuspidatus sequences showed identity with the Austrian isolate CrHo12-650 and the Macedonian ones HoU17 and HoU93; their tuf-type was named "b2" by Aryan et al. (2014) and "ab" by Atanasova et al. (2015). H. obsoletus (Ho-98 sample) and the $R$. panzeri (Rp-202 sample) harboured a tuf-type $\mathrm{b} 1$ corresponding to the classical tuf-type $\mathrm{b}$ according to Aryan et al. (2014).

$\sec Y$ Characterization of the more variable $\sec Y$ gene allowed a finer distinction among the stolbur isolates, 
Table 2 Overview of genetic diversity of FD and BN phytoplasma strains detected in insect bodies collected in vineyards of southern Switzerland

\begin{tabular}{|c|c|c|c|c|}
\hline $\begin{array}{l}\text { Phytoplasma phylogenetic } \\
\text { subgroup }\end{array}$ & Strain $^{\mathrm{a}}$ & Insect species & Insect code & Locality (vineyard code) \\
\hline \multirow[t]{2}{*}{ 16SrV-D } & FD-D & S. titanus & 182,371 & Camorino (436-Caco), Stabio (778-Stab) \\
\hline & FD-D & O. ishidae & 368 & Stabio (778-Stab) \\
\hline \multirow[t]{3}{*}{$16 \mathrm{SrV}-\mathrm{C}$} & FD70-like & O. ishidae & $78,46,370$ & $\begin{array}{l}\text { Lamone (760-Lamo), Stabio, Stabio } \\
\quad(778-S t a b)\end{array}$ \\
\hline & FD-C/FD70-like & O. ishidae & 369 & Stabio $(778-S t a b)$ \\
\hline & FD-C-like & O. ishidae & 63 & Stabio (778-Stab) \\
\hline \multirow[t]{6}{*}{ 16SrXII-A } & tuf-a, seq-a & H. obsoletus & $112,240,271,357$ & $\begin{array}{l}\text { Rancate (912-Ranc), Porza (995-Porz), } \\
\text { Croglio (1195-Crog), Camorino } \\
\text { (436-Caco) }\end{array}$ \\
\hline & tuf-a, seq-b & H. obsoletus & 167,263 & Croglio (1195-Crog) \\
\hline & tuf-a, seq-c & H. obsoletus & 69 & Croglio (1195-Crog) \\
\hline & tuf-b, seq-e & H. obsoletus & 98 & Rovio (1095-Rovi) \\
\hline & tuf-b1, seq-e (=STOL11) & R. panzeri & 202 & Besazio (796-Besa) \\
\hline & tuf-b2, seq-d & R. cuspidatus & 255,264 & Rovio (1095-Rovi), Croglio (1195-Crog) \\
\hline
\end{tabular}

${ }^{\mathrm{a}} \mathrm{FD}$ and FD-like phytoplasmas were codified using: the name of reference strain, if the nt identity was $100 \%$ in all the three genetic regions sequenced; and the suffix "like" if the strain was similar but not identical to reference strain in at least one of the three genetic regions sequenced. Stolbur phytoplasmas were codified using: the tuf-type group (a, b, b1 or b2), and the strain in the $\sec Y$ region (named according to Fig. 2)

that clustered in any case according to their RFLP tuftype. Three different sequence profiles were identified in all the H. obsoletus samples infected by tuf-type a phytoplasma, clearly separated in the phylogenetic tree based on $677 \mathrm{bp}$ from $\sec Y$ amplicons (Fig. 2). In the first one (seq-a), four phytoplasmas isolates from $H$. obsoletus samples shared the same nucleotide identity and they were identical with isolates harboured by $V$. vinifera and $U$. dioica sampled in North Western and North Eastern Italy, respectively. A second sequence (seq-b) comprised just two H. obsoletus samples (Ho167 and Ho-263) and in the third sequence profile (seqc) one H. obsoletus sample (Ho-69) showed a $100 \%$ nucleotide identity with a $V$. vinifera sample from Italy. The two $R$. cuspidatus samples confirmed to harbour identical isolates of ' $C a$. P. solani' and were included in the seq-d profile. In the last sequence profile (seq-e), one H. obsoletus sample showed a $100 \%$ sequence identity with the only one $R$. panzeri sample testing positive in this study, the French STOLC reference strain from Lycopersicum esculentum and STOL11, the classical reference strain of ' $C a$. P. solani'.

In total, five vineyards hosted populations of $H$. obsoletus infected by stolbur phytoplasma and in all sites $C$. arvensis and $U$. dioica were always detected mixed inside the vineyard with the exception of Rovio where $H$. obsoletus were collected on bindweed only. The H. obsoletus population from Croglio harboured all the three genetic variants of tuf-type a, while three local populations (from Rancate, Porza and Camorino) hosted one out of three genetic variants of tuf-type a, and in the last population (from Rovio) a genetic variant of tuf-type b was detected. The two R. cuspidatus samples (Rc-255 and Rc-264) were collected in two different vineyards far away to each other (Rovio and Croglio), despite the phytoplasmas they harboured were identical.

In Table 2 an overview of BN phytoplasma strains detected in this study is reported.

\section{Discussion}

Since outbreaks of grapevine yellows in vineyard can often persist without any clear reasons, the scientific community continues to investigate on the factors that cause these phenomena, while the stakeholders expect immediate answers and effective control strategies.

A detailed knowledge of the epidemiology of phytoplasma-associated diseases is of paramount importance; unfortunately, the epidemiologic cycles can be quite different from one another, owing to factors changing in time and space, such as phytoplasma strains, 


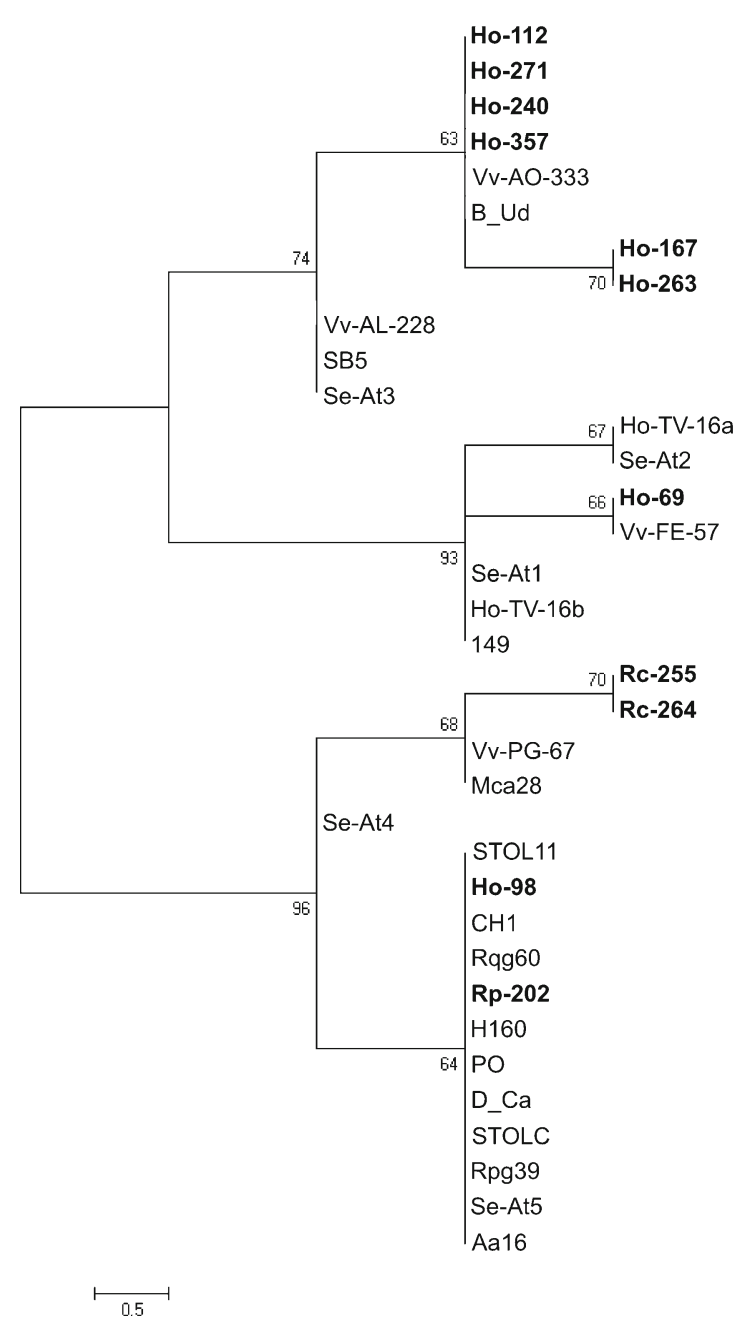

Fig. 2 Phylogenetic tree based on 677 bp of the $\sec Y$ gene sequences obtained from Hyalesthes obsoletus, Reptalus cuspidatus and Reptalus panzeri samples. Swiss samples processed in the current study are evidenced in bold. Bar length is proportional to the number of base substitutions per site. Numbers on the branches are confidence values obtained for 1000 replicates. GenBank

behaviour of insect vectors, primary and secondary hosts, vector population genetics and abiotic factors. In this frame, a well-designed and implemented experimental framework for the investigation of phytoplasma diseases in vineyard is desirable and advantageous to describe the particular characteristics of a territory. That being so, it is widely agreed that the first step is identifying and characterizing the phytoplasmas, since similar or closely related phytoplasmas might exhibit profound differences for host range and vectors, disease expression and development. The second crucial step is linked to the
Country, Region - Municipality

\begin{tabular}{|c|c|c|}
\hline Ho & Switzerland, Tessin - Rancate & \\
\hline Ho & Switzerland, Tessin - Croglio & \\
\hline Ho & Switzerland, Tessin - Porza & \\
\hline Ho & Switzerland, Tessin - Camorino & \\
\hline Vv & Italy, Valle d'Aosta & \\
\hline Ud & Italy, Trentino-Alto Adige - Cembra & \\
\hline Ho & Switzerland, Tessin - Croglio & \\
\hline Ho & Switzerland, Tessin - Croglio & \\
\hline Vv & Italy, Piedmont - Alessandria & \\
\hline Vv & Croatia & \\
\hline Ho & Austria, Burgenland & \\
\hline Ho & Italy, Veneto - Treviso & \\
\hline Ho & Austria, Styria & \\
\hline Ho & Switzerland, Tessin - Croglio & seq-c \\
\hline Vv & Italy, Emilia Romagna - Ferrara & \\
\hline Ho & Austria, Burgenland & \\
\hline Ho & Italy, Veneto - Treviso & \\
\hline Vv & Italy, Marche & \\
\hline Rc & Switzerland, Tessin - Rovio & \\
\hline Rc & Switzerland, Tessin - Croglio & seq-a \\
\hline Vv & Italy, Umbria - Perugia & \\
\hline Vv & Italy, Marche & \\
\hline Vv & Austria, Burgenland & \\
\hline Can & Serbia & 7 \\
\hline Ho & Switzerland, Tessin - Rovio & \\
\hline Vv & Italy & \\
\hline $\mathrm{Rq}$ & Serbia, South Banat & \\
\hline $\mathbf{R p}$ & Switzerland, Tessin - Besazio & \\
\hline Ho & France & \\
\hline Ho & France & $\zeta$ seq-e \\
\hline $\mathrm{Ca}$ & Germany & \\
\hline Le & France & \\
\hline $\mathrm{Rp}$ & Serbia, South Banat & \\
\hline Ho & Austria, Burgenland & \\
\hline Vv & Italy, Abruzzo & \\
\hline
\end{tabular}

accession numbers and details of the reference phytoplasma strains are listed in Supplementary material 2. Host: Ca Convolvulus arvensis; Can Capsicum annuum; Ho Hyalesthes obsoletus; Le Lycopersicum esculentum. Rc Reptalus cuspidatus; Rp Reptalus panzeri; Rq Reptalus quinquecostatus; Ud Urtica dioica; Vv Vitis vinifera

knowledge of biology of insect vectors, their occurrence, their preferred host plants and the spatio-temporal distribution in agroecosystems. The most consistent experimental approach to get objective evidences on occurrence of phytoplasmas and their carriers, according the two above-mentioned steps, is to set up investigations at regional scale, which is also the scale for which effective policy and management measures can be designed and implemented. Moreover, in order to carry out suitable studies concerning aetiology issue of phytoplasma diseases, a multidisciplinary approach is also needed. 
In the present study a multidisciplinary investigation to disentangle the issues of grapevine yellows at regional scale was proposed. We collected leafhopper and planthopper samples from a representative subset of entire winegrowing area South of Swiss Alps, and the results confirmed the adequacy of sampling by means of a multi-method approach (Supplementary Figure 4). As a result, a complete inventory of Auchenorrhyncha inhabiting the vineyards was provided (167 species) and the occurrence of known and potential vectors of phytoplasma was recorded. Similar studies were carried out in North Western Italy and in Austria, where the authors recorded less than one third of the species recorded in this study (32 and 57, respectively; Bosco et al. 1997; Kunz et al. 2010).

In the investigated vineyards five species tested positive for phytoplasmas: $S$. titanus and $O$. ishidae, infected by FD and FD-related phytoplasmas, and $H$. obsoletus, $R$. panzeri and R. cuspidatus, infected by ' $C a$. P. solani'. Among them just two species were abundant and widespread in studied area: the confirmed vector of FD phytoplasma (S. titanus) and a putative vector of BN phytoplasma (R. cuspidatus) (Supplementary Figure 6). $S$. titanus was present with high occurence and abundance only in localities not subjected to mandatory control, wheras we observed very low population abundances in the other Swiss vineyards where insecticides (Buprofezin) were applied twice during the season (Supplementary material 1). Even if a low infection rate was observed at regional scale $(1 \%)$, it is worth highlighting that positive $S$. titanus samples came from a vineyard under mandatory control (one sample, St-371) and from a vineyard where insecticides were not yet been applied, nor symptoms of yellowing in grapevines observed (one sample, St-182). Whilst in Switzerland high rates of FD infected grapevine are still observed locally despite mandatory control programs, we conclude that so far there is no clear relationship between disease outbreaks and S. titanus population density suggesting that other leafhoppers could play a role in spreading the disease.

In Europe, $O$. ishidae was first reported in Switzerland in 2002 (Günthart and Mühlethaler 2002), while in 2010 specimens infected by FD were detected for the first time in Slovenia (Mehle et al. 2010) and then in Italy (Gaffuri et al. 2011). In this study $O$. ishidae infected by FDrelated phytoplasmas was reported for the first time in Switzerland (16SrV-C and D phytoplasma isolates). Interestingly, the most common $16 \mathrm{SrV}-\mathrm{C}$ phytoplasma isolate found in $O$. ishidae Swiss samples was identical with the FD isolate infecting clematis in North-West Italy (Piedmont) and grapevine in South-East France (Savoie) that are regions very close to the Swiss studied area (Fig. 1). In the investigated wine-growing area, $O$. ishidae is quite uncommon and was collected inside vineyards at low density; indeed the mosaic leafhopper inhabits broodleaved forests that surround the studied vineyards and it is strictly associated to woody plants such as Acer, Betula, Carpinus, Crataegus, Malus, Ostrya, Salix and so on (Hamilton 1985). For this reason, O. ishidae populations were detected in the vineyards where the forest is the dominant land unit in the surroundings, and most of the specimens were collected by traps placed along the borders of the forests. An high infection rate was observed both at regional $(27 \%)$ and local scale (ranging from 14 to $100 \%$ ). Five out of six infected $O$. ishidae samples haboured $16 \mathrm{SrV}-\mathrm{C}$ subgroup phytoplasmas, a subgroup that was never recorded before in Switzerland; one sample infected by FD-D isolate was also found, which is the only phytoplasma type detected in grapevine and in S. titanus specimens in this region till now. All these evidences reinforced the hypothesis that $O$. ishidae could play a significant role in spreading different $16 \mathrm{SrV}-\mathrm{C}$ phytoplasma isolates from arboreal plants to grapevine, and FD-D from grapevine to grapevine as well. In a next step, the presence of $16 \mathrm{SrV}-\mathrm{C}$ phytoplasma isolates in grapevine and in wild plant species and the capability of $O$. ishidae to inoculate FD phytoplasmas to grapevine should be verified.

About the cixiids, H. obsoletus was detected with high occurrence. Previous investigations highlighted that $U$. dioica is the preferred host plant of H. obsoletus in most parts of Switzerland (Kessler et al. 2011). Afterwards, in a study on population genetic structure, Maniyar et al. (2013) showed that H. obsoletus populations from southern Switzerland were genetically very similar to those collected in Italy; and the authors highlighted the possibility for the presence of plant-unspecialized $H$. oboletus populations as already reported in Northern Italian winegrowing regions (Imo et al. 2013). In the present study, $H$. obsoletus was collected with relatively low abundance, because its main host can be selectively eliminated inside vineyards due to the recommended removing of the stinging nettle plants (Kehrli and Delabays 2012). In this study, locally $H$. obsoletus reached relatively high densities in vineyards where $C$. arvensis or scattered single individuals of $U$. dioica were recorded. Results reported here confirmed the presence of both tuf-types of $\mathrm{BN}$ 
isolates ("a" and "b") in H. obsoletus, as already observed by Maniyar et al. (2013), and highlighted that the higher infection rates are linked with the "nettle-cycle" based on tuf-type a. Interestingly, in the only vineyard where the host plant of $H$. obsoletus was $C$. arvensis, the vector harboured the tuf-type b1.

Although four species of Reptalus have been recorded in Europe, only $R$. cuspidatus and $R$. panzeri have been detected in southern Switzerland. In the studied region, $R$. cuspidatus was reported as the most common and abundant one in vineyard agroecosystems, whereas the congeneric species was rarely captured inside vineyards and it seems not to prefer grapevine. $R$. panzeri is known to be linked to Rosa spp. and Prunus spp. but also to other woody plants (Clematis, Salix, Crataegus, Pinus, and so on) (Nickel 2003); however, many authors recorded frequently this species on cultivated plants, such as grapevine and maize (Picciau et al. 2008; Jović et al. 2007). Many herbaceous dicotyledons have been recorded as host plants for $R$. cuspidatus, and specimens are frequently captured in Northestern Italian vinegrowing area on the herbaceous dicotyledons Erodium sp., C. arvensis, Echium sp. and Artemisia vulgaris (Picciau et al. 2008). In this study $R$. cuspidatus was abundantly collected on ground floor inside vineyards of mixed-grass vegetation, such as $A$. vulgaris, Geranium rotundifolium, Ranunculus repens and Trifolium spp. Other authors have already reported some of the above mentioned herbaceous plants infected by stolbur phytoplasma, for example A. vulgaris (Credi et al. 2006) and Trifolium pratense (Franova et al. 2009). In the current study, both R. panzeri and $R$. cuspidatus tested positive to tuf-type $\mathrm{b}$ only, and locally they reached high infection rate, up to $100 \%$. Based on our results, the known vector of stolbur $R$. panzeri does not seem to have an important role in the epidemiology of GY phytoplasmas in the investigated area, due to its very low population densities in vineyards. Nevertheless, the detection of a specimen positive to phytoplasma could imply a certain importance for other agroecosystems. Regarding $R$. cuspidatus, it was found to host the tuf-type b2 phytoplasma, previously associated to nettle cycle both in Austrian and in Macedonian vineyards (Aryan et al. 2014; Atanasova et al. 2015). Our R. cuspidatus specimens, however were never collected on nettle in the field. Moreover, the sequencing data showed that the tuf-type b2 identified here is distinct from those already characterized in nettle, H. obsoletus and grapevine samples in the other countries; indeed, it clustered differently in the $\sec Y$ tree compared to the previous works. This suggests that a peculiar tuf-type b2 occurs in Switzerland, and that other weeds may be locally infected with this stolbur isolate, that can be then acquired by $R$. cuspidatus. The range of plants hosting both $R$. cuspidatus and stolbur phytoplasma should therefore be clearly defined; moreover further investigations need to be pursued to clarify the capability of $R$. cuspidatus to inoculate tuftype $b$ phytoplasma to grapevine, as this species represents a risk factor in spreading stolbur phytoplasma in the vineyard agroecosystem.

Although the detection of a GY phytoplasma in an insect body does not necessarily prove its vector status, this detailed screening allowed us to get a clearer vision on the spread of grapevine phytoplasmas in the vineyard agroecosystem at regional scale. This approach is very important for disentangling the complex issue of grapevine yellows, to provide background information to be used to better define the range of alternative and preferred host plants for both vectors and phytoplasmas, and to understand the possible spread of the disease. The main goal must be to develop management strategies aimed at controlling the vectors and the spreading of outbreaks.

Acknowledgments We thank Santiago Schaerer (ACW, Changins, Switzerland) for his help with the DNA extraction and to provide further four positive insect samples. We thank also Mauro Jermini (ACW, Cadenazzo, Switzerland) for useful discussions. Financial support for identification of Auchenorrhyncha was provided by the Dipartimento dell'Educazione della Cultura e dello Sport (DECS) of Ticino Canton.

\section{Compliance with Ethical Standards}

Conflict of interest The authors declare that this work complies to the Ethical Standards of the journal, and that they have no conflict of interest.

\section{References}

Angelini, E., Clair, D., Borgo, M., Bertaccini, A., \& BoudonPadieu, E. (2001). Flavescence dorée in France and Italy occurrence of closely related phytoplasma isolates and their near relationships to Palatinate grapevine yellows and an alder yellows phytoplasma. Vitis, 40(2), 79-86.

Angelini, E., Luca Bianchi, G., Filippin, L., Morassutti, C., \& Borgo, M. (2007). A new TaqMan method for the identification of phytoplasmas associated with grapevine yellows by real-time PCR assay. Journal of Microbiological Methods, 68(3), 613-622. doi:10.1016/j.mimet.2006.11.015. 
Aryan, A., Brader, G., Mortel, J., Pastar, M., \& Riedle-Bauer, M. (2014). An abundant 'Candidatus Phytoplasma solani' tuf b strain is associated with grapevine, stinging nettle and Hyalesthes obsoletus. European Journal of Plant Pathology, 140(2), 213-227. doi:10.1007/s10658-014-0455-0.

Atanasova, B., Jakovljević, M., Spasov, D., Jović, J., Mitrović, M., Toševski, I., \& Cvrković, T. (2015). The molecular epidemiology of bois noir grapevine yellows caused by 'Candidatus Phytoplasma solani' in the Republic of Macedonia. European Journal of Plant Pathology, 142(4), 759-770. doi:10.1007/s10658-015-0649-0.

Batlle, A., Altabella, N., Sabate, J., \& Laviña, A. (2008). Study of the transmission of stolbur phytoplasma to different crop species, by Macrosteles quadripunctulatus. Annals of Applied Biology, 152, 235-242.

Bosco, D., Alma, A., \& Arzone, A. (1997). Studies on population dynamics and spatial distribution of leafhoppers in vineyards (Homoptera: Cicadellidae). Annals of Applied Biology, 130(1), 1-11. doi:10.1111/j.1744-7348.1997.tb05778.x.

Credi, R., Terlizzi, F., Milanesi, L., Bondavalli, R., Cavallini, G., Montermini, A., et al. (2006). Wild host plants of stolbur phytoplasma and its vector, Hyalesthes obsoletus, at sites of grapevine Bois noir occurrence in Emilia-Romagna, Italy. 15th Meeting ICVG, Stellenbosch, SouthAfrica, 182-183.

Cvrković, T., Jović, J., Mitrović, M., Krstić, O., \& Toševski, I. (2014). Experimental and molecular evidence of Reptalus panzeri as a natural vector of bois noir. Plant Pathology, 63(1), 42-53. doi:10.1111/ppa.12080.

Della Giustina, W. (1989). Homoptères Cicadellidae 3: compléments. Faune de France, 73, 1-350.

EPPO (2007). Grapevine flavescence dorée phytoplasma. EPPO Bulletin, 37(3), 536-542. doi:10.1111/j.1365-2338.2007. 01161.x.

Filippin, L., Jović, J., Cvrković, T., Forte, V., Clair, D., Toševski, I., et al. (2009). Molecular characteristics of phytoplasmas associated with Flavescence dorée in clematis and grapevine and preliminary results on the role of Dictyophara europaea as a vector. Plant Pathology, 58(5), 826-837. doi:10.1111/j. 1365-3059.2009.02092.x.

Franova, J., Navratil, M., \& Jakesova, H. (2009). Molecular identification of stolbur phytoplasma associated with red clover dwarf disease symptoms. Journal of Phytopathology, 157, 52-56.

Gaffuri, F., Sacchi, S., \& Cavagna, B. (2011). First detection of the mosaic leafhopper, Orientus ishidae, in northern Italian vineyards infected by the flavescence dorée phytoplasma. New Disease Reports, 24, 22. doi:10.5197/j.2044-0588. 2011.024.02210.1094/Phyto-84-55910.5197/j.2044-0588. 2010.022.011.

Gatineau, F., Larrue, J., Clair, D., Lorton, F., Richard-Molard, M., \& Boudon-Padieu, E. (2001). A new natural planthopper vector of stolbur phytoplasma in the genus Pentastiridius (Hemiptera: Cixiidae). European Journal of Plant Pathology, 107, 263-271.

Gotelli, N. J., \& Colwell, R. K. (2001). Quantifying biodiversity: procedures and pitfalls in the measurement and comparison of species richness. Ecology Letters, 4(4), 379-391. doi:10. 1046/j.1461-0248.2001.00230.x.

Günthart, H., \& Mühlethaler, R. (2002). Provisorische Checklist der Zikaden der Schweiz (Insecta: Hemiptera, Auchenorrhyncha). Denisia, 176, 329-338.
Hamilton, K.G.A. (1985). Leafhoppers of ornamental and fruit trees in Canada. Agriculture Canada Publication, 1779E/F: 1-71.

Holzinger, W. E., Kammerlander, I., \& Nickel, H. (2003). Fulgoromorpha, Cicadomorpha excl. Cicadellidae (Vol. Bd. 1). Leiden: Leiden: Brill.

Imo, M., Maixner, M., \& Johannesen, J. (2013). Sympatric diversification vs. immigration: deciphering host-plant specialization in a polyphagous insect, the stolbur phytoplasma vector Hyalesthes obsoletus (Cixiidae). Molecular Ecology, 22(8), 2188-2203. doi:10.1111/mec.12237.

IRPCM, Phytoplasma/Spiroplasma Working TeamPhytoplasmaTaxonomy Group (2004). 'Candidatus Phytoplasma', a taxon for wall-less, non-helical prokaryotes that colonize plant phloem and insects. International Journal of Systematic and Evolutionary Microbiology, 54, 1243-1255.

Jermini, M., Schaerer, S., Johnston, H., Colombi, L., \& Marazzi, C. (2014). Dix ans de flavescence dorée au Tessin. Revue Suisse de viticulture arboriculture horticulture, 46(4), 222-229.

Johannesen, J., Lux, B., Michel, K., Seitz, A., \& Maixner, M. (2008). Invasion biology and host specificity of the grapevine yellows disease vector Hyalesthes obsoletus in Europe. Entomologia Experimentalis et Applicata, 126(3), 217-227. doi:10.1111/j.1570-7458.2007.00655.x.

Jović, J., Cvrković, T., Mitrović, M., Krnjanjić, S., Petrović, A., Redinbaugh, M. G., et al. (2007). Maize redness in Serbia caused by stolbur phytoplasma is transmitted by. Reptalus panzeri. Bulletin of Insectology, 60(2), 397-398.

Kehrli, P., \& Delabays, N. (2012). Controlling 'bois noir' disease on grapevine: does the timing of herbicide application affect vector emergence? Journal of Applied Entomology, 136(3), 234-237. doi:10.1111/j.1439-0418.2011.01635.x.

Kessler, S., Schaerer, S., Delabays, N., Turlings, T. C. J., Trivellone, V., \& Kehrli, P. (2011). Host plant preferences of Hyalesthes obsoletus, the vector of the grapevine yellows disease 'bois noir', in Switzerland. Entomologia Experimentalis et Applicata, 139(1), 60-67. doi:10.1111/j. 1570-7458.2011.01107.x.

Kunz, G., Roschatt, C., \& Schweigkofler, W. (2010). Biodiversity of panthoppers (Auchenorrhyncha) in vineyards infected by Bois noir phytoplasma. Gredleriana, 10, 89-108.

Landi, L., Isidoro, N., \& Riolo, P. (2013). Natural Phytoplasma Infection of Four Phloem-Feeding Auchenorrhyncha Across Vineyard Agroecosystems in Central-Eastern Italy. Journal of Economic Entomology, 106(2), 604-613. doi:10.1603/ ec12400.

Langer, M., \& Maixner, M. (2004). Molecular characterisation of grapevine yellows associated phytoplasmas of the stolburgroup based on RFLP-analysis of non-ribosomal DNA. Vitis, 43(4), 191-199.

Lee, I. M., Martini, M., Marcone, C., \& Zhu, S. F. (2004). Classification of phytoplasma strains in the elm yellows group (16SrV) and proposal of 'Candidatus Phytoplasma ulmi' for the phytoplasma associated with elm yellows. International Journal of Systematic and Evolutionary Microbiology, 54(2), 337-347. doi:10.1099/ ijs.0.02697-0.

Maixner, M. (1994). Transmission of German grapevine yellows (Vergilbungskrankheit) by the planthopper Hyalesthes obsoletus (Auchenorrhyncha: Cixiidae). Vitis, 33, 103-104. 
Maixner, M., \& Reinert, W. (1999). Oncopsis alni (Schrank) (Auchenorrhyncha: Cicadellidae) as a vector of the alder yellows phytoplasma of Alnus glutinosa (L.) Gaertn. European Journal of Plant Pathology, 105, 87-94.

Maixner, M., Reinert, W., \& Darimont, H. (2000). Transmission of grapevine yellows by Oncopsis alni (Schrank) (Auchenorryncha: Macropsinae). Vitis, 39(2), 83-84.

Maniyar, B., Kehrli, P., \& Johannesen, J. (2013). Population structure and incidence of the stolbur phytoplasma vector Hyalesthes obsoletus (Cixiidae) among geographic regions in Switzerland. [Article]. Journal of Applied Entomology, 137(8), 589-600. doi:10.1111/jen.12034.

Marcone, C. (2014). Molecular biology and pathogenicity of phytoplasmas. Annals of Applied Biology, 165(2), 199-221. doi:10.1111/aab.12151.

Martini, M., Botti, S., Marcone, C., Marzachì, C., Casati, P., Bianco, P. A., et al. (2002). Genetic variability among Flavescence dorée phytoplasmas from different origins in Italy and France. Molecular and Cellular Probes, 16, 197-208.

Mehle, N., Seljak, G., Rupar, M., Ravnikar, M., \& Dermastia, M. (2010). The first detection of a phytoplasma from the $16 \mathrm{SrV}$ (Elm yellows) group in the mosaic leafhopper Orientus ishidae. New Disease Reports, 22, 11. doi:10.5197/j.20440588.2010.022.01110.1111/j.1365-3059.2009.02092.x10. 1111/j.1365-3059.2007.01688.x.

Mikec, I., Križanac, I., Budinščak, Z., Šeruga Musić, M., Krajačić, M., \& Škorić, D. (2006) Phytoplasmas and their potential vectors in vineyards of indigenous Croatian varietis. In Meeting of the International Council for the Study of Virus and Virus-like Diseases of the Grapevine (ICVG), Stellenbosch (South Africa), (pp. 255-257)

Nickel, H. (2003). The Leafhoppers and Planthoppers of Germany (Hemiptera Auchenorrhyncha): Patterns and strategies in a highly diverse group of phytophageous insects: Copublished by Pensoft Publishers. Keltern: Sofia-Moscow and Goecke \& Evers.
Palermo, S., Elekes, M., Botti, S., Ember, I., Alma, A., Orosz, A., et al. (2004). Presence of stolbur phytoplasma in Cixiidae in Hungarian Vineyards. Vitis, 43, 201-203.

Picciau, L., Lessio, F., \& Alma, A. (2008). Preliminary data on the Cixiid fauna of the vineyard agro-ecosystem in Piedmont (North-Western Italy). Bulletin of Insectology, 61(1), 197-198.

Pinzauti, F., Trivellone, V., \& Bagnoli, B. (2008). Ability of Reptalus quinquecostatus (Hemiptera: Cixiidae) to inoculate stolbur phytoplasma to artificial feeding medium. Annals of Applied Biology, 153, 299-305.

Ribaut, H. (1936). Homoptères Auchènorhynches 1. (Typhlocybidae). Faune de France, 31, 1-228.

Riedle-Bauer, M., Sára, A., \& Regner, F. (2008). Transmission of a Stolbur Phytoplasma by the Agalliinae LeafhopperAnaceratagallia ribauti(Hemiptera, Auchenorrhyncha, Cicadellidae). Journal of Phytopathology, 156(11-12), 687-690. doi:10.1111/j.1439-0434.2008.01416.x.

Schaerer, S., Johnston, H., Gugerli, P., Linder, C., Schaub, L., \& Colombi, L. (2007). Flavescence doree in Switzerland: spread of the disease in canton of Ticino and of its insect vector, now also in cantons of Vaud and Geneva. Bulletin of Insectology, 60(2), 375-376.

Schmid, A., \& Emery, S. (2001). La maladie du Bois noir dans le vignoble valaisan. IOBC/wprs Bulletin, 24, 287-289.

Schneider, B., Gibb, K. S., \& Seemuller, E. (1997). Sequence and RFLP analysis of the elongation factor tu gene in differentation and classification of phytoplasmas. Microbiology, 143, 33813389.

Schvester, D., Carle, P., \& Moutous, G. (1963). Transmission de la flavescence dorée de la vigne par Scaphoideus littoralis Ball. Annales des Epiphyties, 14, 175-198.

Sforza, R., Clair, D., Daire, X., Larrue, J., \& Boudon-Padieu, E. (1998). The role of Hyalesthes obsoletus (Hemiptera: Cixiidae) in the occurrence of bois noir of grapevines in France. Journal of Phytopathology, 146(11-12), 549-556.

Trivellone, V., Schoenenberger, N., Jermini, M., de Bello, F., Mitchell, E. A. D., \& Moretti, M. (2014). Indicators for taxonomic and functional aspects of biodiversity in the vineyard agroecosystem of Southern Switzerland. Biological Conservation, 170, 103-109. 